\title{
Correspondence
}

To the Editors:

\section{Use of exclusive breast feeding definitions; a call for action!}

\author{
Sri Lanka Journal of Child Health, 2009; 38: 77-78
}

(Key words: Exclusive breastfeeding, definitions, 24 hour recall method, strict definition)

\begin{abstract}
We read the article titled "Influence of exclusive breast feeding on occurrence, severity and recurrence of acute lower respiratory infections in preschool children" published in the Sri Lanka Journal of Child Health ${ }^{1}$ with great interest. The observation made on the duration of exclusive breastfeeding and the low rates of six months exclusive breastfeeding was very important in our context.
\end{abstract}

However, we observed that one major definition is not reported in the paper. Being a case-control study, defining the exposure under investigation is of utmost importance. The exposure studied in this paper was Exclusive Breast Feeding (EBF). But, the definition used for enumeration of EBF has not been reported in the article. The study sample included only 16 cases who were within the first six months of life. Majority were more than one year of age. Neither the most widely used WHO criteria $^{2}$, nor the strict definition of breastfeeding according to Labbock criteria ${ }^{3}$ seems to have been used in this study. A literature review on Sri Lankan breastfeeding studies also revealed that EBF is inadequately defined in most studies.

We would like to present the most widely used definitions for enumeration of EBF in clinical and epidemiological studies.

The EBF definition recommended by the WHO for research purposes is based on the 24 hour dietary recall method. Investigators are expected to obtain a detailed history of feeding during past 24 hours, preferably using an itemised check list containing commonly used local complementary foods. This method can be used only for infants of 0-6 months of age. Children more than six months of age should not be assessed using this definition, since it is recommended to start complementary feeds after the completion of sixth month. This is the method used in the Sri Lanka Demographic and Health Survey (DHS). The reported EBF rate of $75 \%{ }^{4}$ in Sri Lanka was based on this method.

The Labbock strict definition of breast feeding defined the EBF rate as "the proportion of infants less than six months of age who received only breast milk". For this definition, either the study should be a cohort study or the investigator should obtain a detailed history of feeding practices since birth. This is not an easy task. However, we observed in our earlier studies that the use of an event calendar will yield the best results ${ }^{5-8}$. This method will not provide valid estimation for EBF if the study sample consisted of infants more than one year of age. An analysis on recall bias on duration of EBF showed that longer the duration of recall, higher will be the estimated EBF rate.

For studies where the study sample consists of children aged 1-5 years of age, defining duration of breastfeeding is difficult. Recall bias play a huge role in this definition making the results unreliable. Therefore strict standardization of procedures, minimizing observer variability and using more stringent criteria for classification is essential to improve the validity of results.

We invite all investigators to use valid definitions of EBF in their studies, and to report the methods used, so that the results would be valid and comparable with results of studies done elsewhere. This would provide supportive evidence to further strengthen and to improve the infant care service provision in Sri Lanka.

\section{References}

1. Pathirage SN, Pathigoda S, Peiris R, Sri Ranganathan S. Influence of exclusive breast feeding on occurrence, severity and recurrence of acute lower respiratory infections in preschool children. Sri Lanka Journal of Child Health, 2009;31:21-24.

2. World Health Organization. Indicators for assessing infant and young child feeding practices : Part 1 Definittions. Geneva, Swizterland: World Health Organization, 2008.

3. Labbok M, Krasovec K. Toward consistency in breastfeeding definitions. Stud Fam Plann 1990;21(4):226-30.

4. Department of Census and Statistics. Sri Lanka Demographic and Health Survey 2006/7 Preliminary Report (Draft). Department of Census and Statistics In collaboration with Ministry of Health care and Nutrition. Colombo, 2008. 
5. Agampodi SB, Agampodi TC. Effect of low cost public health staff training on exclusive breastfeeding. Indian $J$ Pediatr 2008;75(11):1115-1119.

6. Agampodi SB, Agampodi TC, Piyaseeli UK. Breastfeeding practices in a public health field practice area in Sri Lanka: a survival analysis. Int Breastfeed J 2007;2:13.

7. Agampodi SB, Fernando S, Dharmaratne SD Comparison of definitions used for Exclusive Breast Feeding-The Labbock's strict definition and the WHO definition as methods of estimating Exclusive Breastfeeding rates in Sri Lanka. Ceylon Medical Journal 2009;54 suppl 1):20.

8. Agampodi SB, Fernando S, Dharmaratne SD. Prevalence of undesirable infant feeding practices in a rural community in Matale district- a preliminary analysis Ceylon Medical Journal 2009;54 suppl 1:57.

\section{Dr. Suneth B Agampodi \\ Dr. Thilini Agampodi}

Department of Community Medicine, Faculty of Medicine and Allied Science, Rajarata University of Sri Lanka, Saliyapura

\section{Reply by the authors:}

We are very happy that our article on "Influence of exclusive breast feeding on occurrence, severity and recurrence of acute lower respiratory infections in preschool children" has initiated a discussion on a very important subject. We thank the authors of "Use of exclusive breast feeding definitions; a call for action!" for their keen interest in this most important aspect of infant care.

We agree that for a case control study, definition of exposure and definition of outcomes are very important. Case control study has its inherent limitation of recall bias as the data are generally collected retrospectively.
Our study focused on preschool children and information on the duration of exclusive breast feeding was obtained from mothers. We used the Labbock strict definition of breast feeding and duration of exclusive breast feeding was defined as "time period during which a baby received only breast milk". We agree that recall bias would have influenced the rate of exclusive breast feeding in infants over one year. However, we took all possible precautions to minimise the recall bias such as adequate explanation, allowing adequate time for the mother to recall, avoiding prompting by the investigators and using the same investigator in interviewing all the mothers. This Standard Operational Procedure (SOP) was followed for both cases and controls.

Recall bias overestimates the rate of exclusive breast feeding. Certain extent of overestimation could always occur despite the SOP. In our study, we have compared the rate of exclusive breast feeding between cases and controls. Recall bias and overestimation would have equally occurred in both groups without blunting the final difference between the two.

Our results showed that $53 \%$ of cases were exclusively breast fed for four or more months compared to $68 \%$ of controls. The real values could be still lower.

\section{Dr. Shalini Sri Ranganathan}

Senior Lecturer in Pharmacology and Consultant Paediatrician, Faculty of Medicine, University of Colombo 